\title{
Development of Korean Style Loungewear (Part II)
}

\author{
Keum-Seok Chae $\cdot$ Yooshin $\mathrm{Na}^{\dagger}$ \\ Dept. of Clothing \& Textiles, Sookmyung Women's University
}

Received July 17, 2012; Revised (September 28, 2012; November 19, 2012); Accepted November 19, 2012

\begin{abstract}
The paper details the development of a Korean style loungewear based on traditional Korean hanbok clothing. For this, we surveyed and collected data from 3,500 adults over 20 years of age. The questions were on their preferences of hanbok for both traditional and contemporary styles as well as loungewear. The survey showed that preferences for hanbok were high for both traditional and contemporary styles. The survey also suggests how a successful mass-produced Korean style loungewear should be designed to increase its popularity. It should feature comfortably simplified silhouettes, a selection of contemporary colors from Korean symbolic colors, modernized Korean textile surface designs, and easy-to-maintain healthy fabric. Based on these results, the development direction of Korean style loungewear was set and various loungewear samples were produced. Another group of survey participants were asked to try on samples and their preferences were surveyed. At the conclusion, a version that was most preferred among the participants was obtained. We suggest one-piece style and two-piece style designs with silhouettes based on the characteristics of traditional Korean costumes. The preferred fabric for loungewear was natural and soft cotton. We suggest the necessity and effectiveness of loungewear made with environment-friendly hanji-fabric. The main colors for loungewear were selected based on survey: blue, red, white, and saekdong (color stripes). We made color combinations with the main colors then applied the textile surface designs. The traditional Korean patterns of Hangeul (Korean letters), taegeuk (yin-yang), geometric figures, and samjogo (three-legged crow) were also chosen, and then loungewear using the textile designs was developed. The results have been shown on the "Han Style Fashion Show" by Jeonju City and exhibited in the "Seoul Living Design Fair" and the "International Art \& Craft Trend Fair" in COEX, Seoul.
\end{abstract}

Key words: Korean style, Loungewear, Hanbok, Cultural commodity

\section{Introduction}

Development of Korean style loungewear based on traditional Korean clothes could be able to help advance the identity of Korean culture in the world. The advancement of Korean style loungewear could provide opportunities to experience Korean culture, which will help promote Korean image and help the Korean tourist industry.

${ }^{\dagger}$ Corresponding author E-mail: ysn86@hotmail.com

This research was financially supported by Sookmyung Women's University in 2012.
Hence, this paper describes an attempt to modernize and globalize the traditional Korean costume. In an effort to broaden the image of traditional Korean clothes among the general population, we conducted a survey and then determined the direction of designing loungewear with images and styles that reflect Korean cultural strengths; with the survey results, actual samples were designed.

The world's successful fashion designer brands share one point - modernization of tradition (Chae, 2006). A brand image is strengthened when it achieves (1) characteristics unique to its own culture and (2) universality so that it is accepted throughout the

(C) 2012, The Korean Society of Clothing and Textiles. All rights reserved. 
world. Provided this, it should be noted that eastern clothing has been given attention as a distinct set of styles in the fashion industry where western styles are the norm.

China has successfully transformed chipao, traditional Chinese clothing, as one of China's popular cultural commodities. Chipao kept its unique Mandarin collar and long-slit skirt while modernizing its silhouette, fabric, color, and pattern. Japan has also been successful in adapting kimono for everyday life and spreading its wear in the general population. Yukata, a simplified version of kimono, is now a tourist item that delivers a strong image of Japan to foreign tourists in Japan's hotels, hot springs, or their traditional inns (Chae, 2007).

Loungewear provides comfort for effective rest. The importance of resting cannot be overemphasized in today's world, and development of loungewear is a natural response to this trend. If international hotels in Korea adopt loungewear of a unique Korean style, it could be a special boon to their marketing towards foreign guests.

In the $<$ Part I $>$ of the thesis ${ }^{『}$ Development of Korean Style Loungewear $₫$, we discussed about the results of the survey and the development of loungewear in silhouette. Now in $<$ Part II $>$, we will deal with the development of loungewear in colors and patterns, and show the complete design results.

\section{Theoretical Framework}

\section{Loungewear}

Before the development of Korean style loungewear, the meaning of 'loungewear' should be defined. 'Lounge' means 'living room' or sitting room as a noun, and it also means 'relax' as a verb. Therefore, the word 'loungewear' means a kind of clothing that is designed for resting to relax at home or in hotels. It could be worn at home when he or she is alone at home or when close friends visit his or her houses. It could also be worn in the hotel lobby as well as in hotel rooms. Loungewear should provide comfort for effective rest, but it is different from pajamas for only sleeping at night. 'Lounging robe', 'room wear', 'dressing gown', and French words 'robe de chambre' are also used for similar meaning.

The styles of loungewear are diverse depending on the culture, area and time. However, they have something in common that are designed for comfort resting. They are usually roomy for easy putting on and taking off. Most of loungewear is made without buttons and zippers, because they could disturb sleep. Instead, they use fabric closure like ribbons or strings. Some loungewear is big enough or stretchable enough not to need closure like buttons and zippers. The fabrics used for loungewear are usually soft and sometimes stretchy (Fashion dictionary compilation committee, 1999).

\section{Korean Traditional Clothing}

Korea's representative traditional clothing is divided into jeogori, baji, chima, and po, which constitute the complete hanbok attire. These separate basic categories are similar in other Asian cultures. However, their structures, configurations, and embedded philosophy are unique to Korea.

\section{1) Jeogori (Traditional Korean Jacket)}

Jeogori is a kind of traditional Korean upper-wear, and its purpose is the same as jackets of the western world. Jeogori successfully characterizes traditional Korean clothing, consisting of gil (bodice), git (collar), seop (attached cloth on the front of bodice), and somae (sleeve), which all show geometric figures of rectangles and triangles. An-git (inward collar) and geot-git (outward collar) form a slanted V-shaped neckline. These collars meet in the central front, drawing a curve and giving a solid impression. Goreum, Korea's unique tying method using two attached pieces of cloth, looks dynamic, as if it is dancing as the wearer's body moves. Since the Goryeo Dynasty (9181392), a triangular piece of cloth called mu has been added to the side of jeogori bodice, and this has the effect of widening the lower part of jeogori, making an A-lined silhouette (Chae, 2006). The whole structure of jeogori is symmetric, but the details of jeogori 
such as seop (attached cloth on the front of bodice) and goreum are asymmetric.

\section{2) Baji (Traditional Korean Pants)}

In ancient Korea, baji (traditional Korean pants) was worn by both men and women. It has remained as men's pants, but for women it developed into innerwear worn under skirts. Having been heavily influenced by horse-riding nomads from the northern region of the Korean peninsula, Korean baji has a unique configuration from those of the western pants.

In traditional Korean baji, marupok (outer leg part of baji) is rectangular and sapok (inner leg part of baji) consists of two differently sized triangles. And the rectangular waistline forms a circular shape. The configuration features spatial flexibility in modifying clothes depending on the wearer's body shape. Korean baji is voluminous. The lower ends of baji, however, are tied firmly with additional cloth ties or bands making the ankle parts fit for adept body movements. Also, the leg parts are spacious for comfortable body movements.

\section{3) Chima (Traditional Korean Skirt)}

Chima, traditional Korean skirt for women, is put on by wrapping the lower part of the body like a wrap skirt. Chima is made of three pieces of long rectangular fabric and is gathered at the waist; it allows for flexible body movements when worn since its shape, which is like the elliptical silhouette of traditional Korean pottery, is quite voluminous.

\section{4) Po (Formal Traditional Korean Outerwear)}

$P o$ is traditional Korean unisex outerwear which is similar to the coat of the western world and it completes attire. This basic form of clothing has long existed, and it has developed into winter-clothes, street clothes, and ceremonial clothes, respectively (Chae $\&$ Koh, 2009). During the Joseon Period (1392-1897), which stressed strict manners of wearing clothes according to social status, po was seen to show ideology and aesthetics. Po is characterized by the fact that it consists of rectangular shapes and resembles a bird's body, and it is an extension of the traditional jacket, or jeogori.

\section{Survey \& Data Analysis}

\section{Methods}

Five thousand men and women over the age of 20 in Korea were asked to answer a survey form during the period of August 1 and October 31, 2007, and 3,500 replies were received. The questionnaire is composed of questions about traditional hanbok, current fashion that characterizes Korea, and effect of development of Korean style loungewear. Design preferences of Korean style loungewear were also asked. SAS SYSTEM was used for obtaining various statistics including frequency and percentage.

\section{Data Analysis}

\section{1) Public Response on Traditional Korean Cos- tume \& Korean Style Fashion}

This survey shows $89 \%$ of the whole respondents had favorable opinions on traditional hanbok in the market. However, the respondents also thought that traditional hanbok is not suitable for modern lifestyle. The majority of our respondents answered that they favor Korean style fashion but are hesitant to try (57.8\%), or they favor and wish to wear (19.9\%). Making clothes more affordable and adding functionality will help make Korean style fashion appeal more to potential customers.

\section{2) Public Response on Korean Style Loungewear}

As for the effect of developing Korean style loungewear, $82.5 \%$ of our respondents said its growth would have promotional effect for tourism, providing a strong justification for developing one such a model. It should also be noted that $96.1 \%$ of our respondents indicated meaningful interest in loungewear of Korean style. Public opinion looks favorably on the development of Korean style loungewear. <Table 1> summarizes the results of our survey on traditional Korean costume, Korean style fashion, and loungewear of Korean style.

According to the survey on design preference of Korean style loungewear, $40.4 \%$ of all our respondents favored one-piece style, $23 \%$ liked two-piece 
Table 1. Response on traditional Korean costume, Korean style fashion and Korean style loungewear

\begin{tabular}{c|c|c|c}
\hline \hline Category & Perception & Results & $\%$ \\
\hline \multirow{4}{*}{ Traditional Korean Costumes } & Positive & Very interested / Favorable & 89.0 \\
\cline { 2 - 4 } & Negative & $\begin{array}{c}\text { Inconvenient: Body movement / maintenance / price } \\
\text { Unaesthetic: design and fabric need improvement } \\
\text { Not for the general public: ready-made clothes not available. }\end{array}$ & 11.0 \\
\hline \multirow{2}{*}{ Korean Style Fashion } & Positive & Highly favorable, interested & 77.7 \\
\cline { 2 - 4 } & Negative & $\begin{array}{c}\text { Uncomfortable, restricted body movement, price } \\
\text { Outdated and cheap-looking design }\end{array}$ & 22.3 \\
\hline \multirow{2}{*}{ Korean Style Loungewear } & Positive & Boosting Korea's image & 82.5 \\
\cline { 2 - 4 } & Negative & None & 17.5 \\
\hline
\end{tabular}

style, while $35.5 \%$ liked both styles. The respondents believe that git (the collar) and goreum (the tying method) are the unique aspects of the traditional clothes that best represent Korean style $(47.8 \%$ and $44.4 \%$ respectively, multiple selections were allowed). As for length of one-piece style loungewear, $52.3 \%$ of the respondents preferred calf-length, $34.7 \%$ kneelength, and $13.0 \%$ ankle-length. As for skirt length, $48.5 \%$ preferred calf-length; $30.0 \%$ liked knee-length, and $21.5 \%$ ankle-length. For the pants, $54.9 \%$ preferred the length to end at the ankles, $28.5 \%$ the calves; $16.5 \%$ the knees.

As for the fabric of choice, $51.3 \%$ of the respondents selected cotton due to its natural origin and softness (Table 2). $68.0 \%$ of the respondents showed interest in clothes made of hanji-fiber which has bugrepellant and far-infrared ray radiation properties.

According to the survey on color preference, red was thought to best represent Korea by $43.2 \%$ of the
Table 2. Fabric preferred for Korean style loungewear

\begin{tabular}{c|c|c}
\hline \hline Fabric & Count & $\begin{array}{c}\text { Percentage } \\
(\mathbf{\%})\end{array}$ \\
\hline Hanji-fabric & 605 & 17.5 \\
\hline Towel / Flannel & 364 & 10.5 \\
\hline Cotton & 1777 & 51.3 \\
\hline Glossy fabric (Silk/Silky fabric) & 476 & 13.7 \\
\hline Jersey knit & 245 & 7.1 \\
\hline Number of respondents & 3467 & 100.0 \\
\hline
\end{tabular}

respondents. $42.7 \%$ said saekdong (Korean color stripes), $30.7 \%$ white, $14.7 \%$ blue, and $13.8 \%$ purple (Table 3). Those in their 20 s preferred red, and those older than the age of 30 preferred saekdong. Students and hoteliers preferred red while housewives, professionals, workers in the service field preferred saekdong. For Korean style loungewear, however, 36.2\% of the respondents thought that white was the color most suitable. $24.3 \%$ thought blue was, $17.7 \%$ saek-

Table 3. Preference for Korean colors (multiple answers)

\begin{tabular}{c|c|c|c|c}
\hline \hline \multirow{2}{*}{ Color Group } & \multicolumn{2}{|c|}{ Colors for Korean Image } & \multicolumn{2}{c}{ Colors for Korean Style Loungewear } \\
\cline { 2 - 5 } & Count & Percentage (\%) & Count & Percentage (\%) \\
\hline White & 1068 & 30.7 & 1255 & 36.2 \\
\hline Black or Grey & 340 & 9.8 & 487 & 14.1 \\
\hline Red & 1502 & 43.2 & 587 & 17.0 \\
\hline Yellow & 431 & 12.4 & 584 & 24.3 \\
\hline Blue & 512 & 14.7 & 843 & 14.5 \\
\hline Green & 421 & 12.1 & 503 & 15.8 \\
\hline Purple & 475 & 13.8 & 546 & 9.9 \\
\hline Brown & 173 & 5.0 & 614 & 17.7 \\
\hline Saekdong & 1483 & 42.7 & & 3463 \\
\hline
\end{tabular}


dong, $17.0 \%$ red, and $16.9 \%$ brown. Those in their 20 s preferred blue colors; those in their 30 s and above preferred white.

Based on the survey, the traditional patterns that best represent Korean style are taegeuk (42.6\%), nature symbols (39.0\%), signs of luck (28.3\%), geometric patterns $(27.2 \%)$, and Hangeul (Korean letters; $24.7 \%$ ) (Table 4). A wide variety of Hangeul calligraphy is also preferred as a Korean pattern.

\section{Design Development of Korean Style Loungewear}

This design development consisted of four major stages. The stages are silhouette design development, textile selection, color development and textile surface design development. The questionnaire on development of loungewear of Korean style, too, consisted of questions on silhouette, detail, textile, color, and fabric pattern.

The foundation of the silhouettes was the characteristic geometric structures of traditional jeogori, chima, baji, and po. Functionality-oriented fabrics were listed. Colors that represent Korea were deduced based on our survey and other studies. Pre- ferred traditional patterns were simplified and modernized to develop various patterns. DTP method was used for printing.

\section{Silhouette Design Development}

\section{1) One-Piece Styles}

The characteristic design details are git (Korean neckline) and goreum (Korean tie ribbon) that best represent Korean style. Depending on the adjustment method, we suggested 'opened style' and 'closed style' designs. Various adjustment methods were tried: a 'belt style', 'short-goreum style', 'long-goreum style' and 'button style' were developed. We suggested 'regular style' designs that have a form of extended upperwear, and 'yoke style' designs that have a horizontal seam line between the upper and lower parts.

For git (collar), we suggested a long 'chest-lengthcollar' and a short 'neck-length-collar.' For sleeves, a 'straight-line sleeve' and a 'round sleeve' were developed.

\section{2) Two-Piece Styles}

For two-piece styles, two different styles were developed, an opened style that has adjustable goreum

Table 4. Korean traditional patterns used for the survey

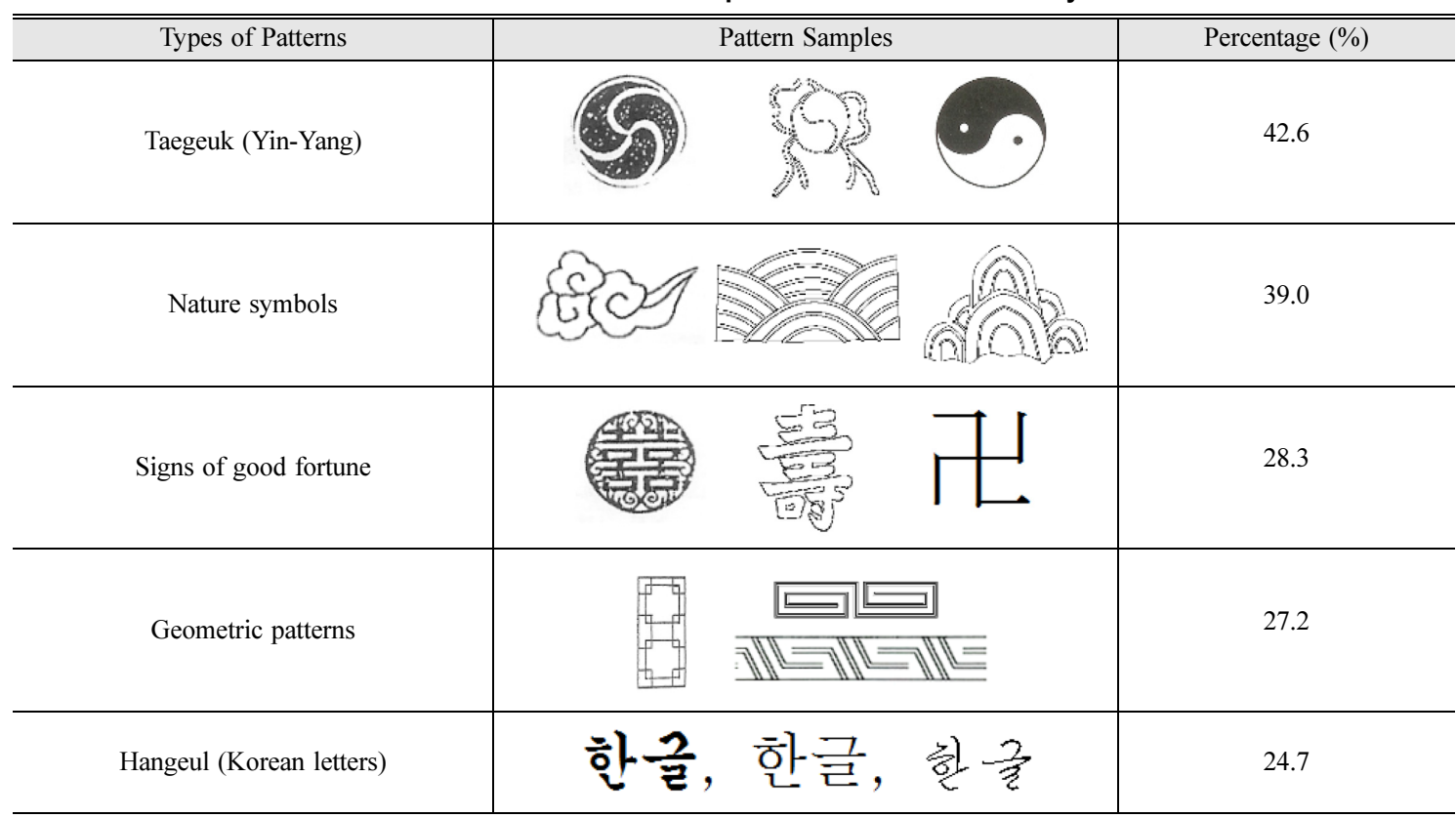


and a closed style that resembles a comfortable tshirt. For the two-piece styles, chima (skirts) were developed for women and baji (pants) for men.

Each of the one and two piece designs underwent fitting tests by twenty researchers. $<$ Table $5>$ summarizes the results of silhouette designs for Korean style loungewear.

\section{Fabric Selection}

As for the fabric of choice, cotton was most preferred due to its natural origin and softness. It should

Table 5. Silhouette designs of Korean style loungewear

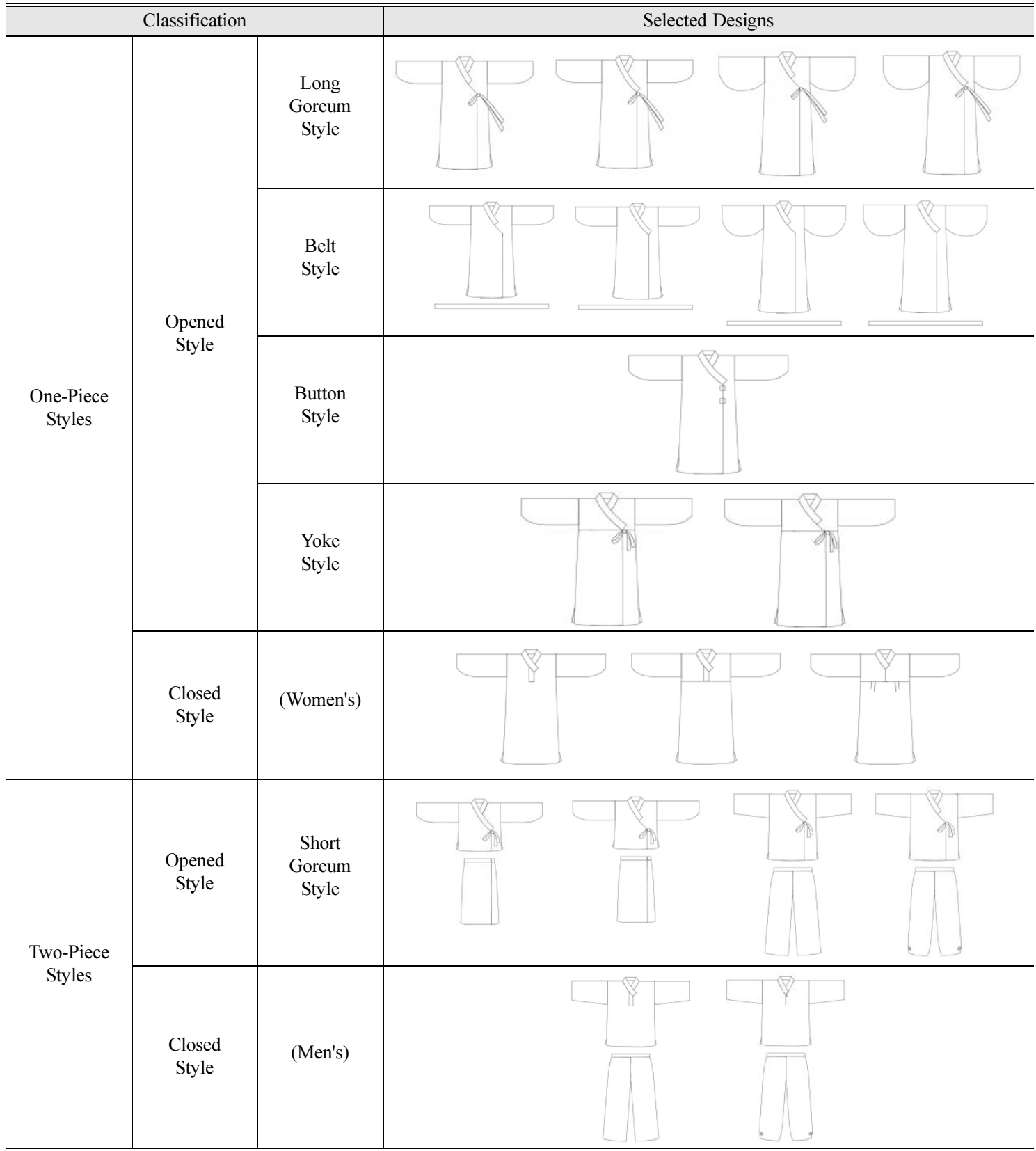


be noted that Chae (2001) also came to the same conclusion - tourists and workers in the tourism industry both preferred cotton.

The world's consumer culture is changing and new industries are emerging as the trend of healthy living spreads. Its influence on the clothing industry is apparent as new kinds of fabric come with healthaware functionality, and clothes for sports, leisure and outdoor activities increase in popularity. Especially, fabrics with health-aware functionality have been developed actively since the market demand for such products has increased.

Of our respondents, $68.0 \%$ showed interest in clothes made of hanji-fiber which has bug-repellant and far-infrared ray radiation properties. This makes production of hanji-fiber clothes not only needful but also practical. Hanji-fiber fabric interweaves hanji and cotton, $50.0 \%$ each. Hanj-fiber is made from traditional Korean paper made from mulberry trees and is a popular environment-friendly material: it removes odor, is antibiotic, and has excellent durability. It is easy to dye and wash, and lighter and stronger than cotton.

\section{Color Development}

Colors were chosen based on the responses to the design preference survey of Korean loungewear and other similar studies conducted previously. According to the survey, the preferred color groups that best represent Korea were red, saekdong (Korean color stripes), white, blue, and purple. Their ideas differed among age groups and professions. However, the colors the respondents thought most suitable for Korean style loungewear were white, blue, saekdong, red, and brown. According to the results, Korean style loungewear should be designed mainly in these colors: blue, red, white, and saekdong. According to Chae's (2001) study, $77.7 \%$ of the respondents preferred colors of pale tone. It was also discovered that those who were more interested in Korean images also tended to prefer pale tones. Survey results indicate that pale tones should be the main colors for loungewear. With these survey data and Chae's (2011) previous study, we decided the colors suitable for
Korean style loungewear: white is most preferred among Koreans and considered most representative of Korean culture. Next, red and blue colors were favored. The next preferred colors were saekdong, purple, yellow, green, black or gray, and brown.

Based on the results of the survey, six groups of color combinations are proposed as follows. Every color group was tried to be used with white background color which is the most preferred color.

The first group is 'Blue Group' which is the combination of Korean blue and jade. Among blue-tone colors, pale-blue jade color is suggested as a main color for loungewear. Jade is the color of Goryeo celadon, which is one of Korea's representative cultural items, so the color jade embodies Korea. Korean blue (indigo blue) which is often found in traditional Korean costumes is also proposed as a representative Korean color.

The second group is 'Green Group' which is the combination of green and jade. Jade, the color of light blue mixed with a small amount of green, is suggested to be used with green to design fabric patterns.

The third group is 'Yellow Group' which is the combination of olive green and yellow. The suggested combination color is olive green. Red clay, another of Korea's representative colors which is close to brown, is also used in combination.

The fourth group is 'Red Group' which is the combination of red and pink. A combination of red and pink is suggested, because red is a highly preferred color to represent Korea. Pink is a less saturated color of red and it has a relaxing effect.

The fifth group is 'Black \& White Group' which is the combination of black and white. White, the most preferred color for Korean imagery and Korean style loungewear, is suggested to be combined with black. The combination of black and white encompasses the feeling of traditional Korean calligraphy and also has a modern feeling.

The sixth group is 'Saekdong Group (Color Stripes)'. Saekdong, which is a striped combination of various primary colors, is one of Korea's representative color combinations often used for clothing. Saekdong could be too bold for loungewear, so it should 
be mixed with white or black.

\section{Textile Surface Design Development}

Based on the survey, the traditional patterns that best represent Korean style are taegeuk, nature symbols, signs of luck, geometric patterns, and Hangeul (Korean letters). Taegeuk, which symbolizes the yin and the yang, is also a symbol of Korea. Nature symbols show clouds, waves, and mountains. Lucky symbols are ancient letters that mean happiness, longevity, or good fortune. Geometric patterns that have long been used for traditional Korean crafts are also included. A wide variety of Hangeul calligraphy is also preferred as a Korean pattern.

Our survey indicated that Korean letters are not the most internationally well known, yet Hangeul has been getting increased attention worldwide. It also is meaningful to develop the letters '전주한지 (Jeonju Hanji)' which stands for hanji paper fabric made in the city of Jeonju; thus logos that use the characters

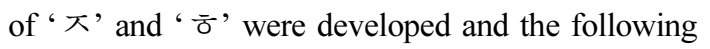
pattern is proposed. Using modern fonts, combinations of the letter 'ㅈ' and ' $\bar{~}$ ' from the word '전주 한지' were laid horizontally or vertically, while overlapping or rotating. Patterns were developed in this way; each of which was laid out of one direction or two directions; symmetric, tilted, or crossed.

The traditional Korean patterns of taegeuk (yinyang), geometric figures, and samjogo (a three-legged crow) were also chosen for textile designs. The threelegged crow figure especially has recently gained popularity among Koreans. Some Korean ink-and-wash art drawings are used for textile designs. To avoid monotony, some of them were combined with the Korean letter patterns.

Various patterns were developed based on the survey, and then the preferred colors were applied to the patterns. Among the textile surface designs developed, the well-designed results which were suitable for the loungewear were selected. Twenty researchers participated in the selection of the textile surface designs.

Each of these patterns combined with the four color combinations is shown in $<$ Table $6>$. The de- signs were printed on hanji-fabric and dyed using the DTP method.

The combined aspects of silhouette, color, and patterns are shown in $\langle$ Table $7>$. The results were produced in samples and their patent is pending in the patent office as of 2008 .

\section{Conclusions and Implications for Future Research}

The summary of this study is as follows:

First, preference of traditional hanbok among the respondents is found to be high, but its hindrance to body movement and difficult maintenance make hanbok's place in society less favorable. If the silhouette is modified to allow freer body movement and a different fabric is used to make maintenance easier, the majority are willing to buy hanbok.

The majority of the respondents favored the fashion of traditional Korean style, but this preference does not translate to actual purchases due to expensive prices and uneasy body movements when wearing hanbok. Hanbok is regarded as a costume worn only for special occasions such as traditional holidays or ceremonies. Hence, to popularize hanbok, the prices need to be more affordable and the fabric and silhouette need to be made more comfortable. It was found that development of Korean style loungewear was considered an effective way to promote Korean images.

Second, we designed an assortment of loungewear based on the responses of the survey. We suggest one-piece type and two-piece type designs with silhouettes based on the characteristics of traditional jeogori (jacket), chima (skirt), baji (pants), and po (coat). The upper part designs of the one-piece and two-piece types come in both traditional open-styles and modified closed-styles; the lower-wear pieces come in chima and baji. The preferred fabric for loungewear was cotton, which is natural and soft. The latest trend among consumers is health-consciousness; which calls for development of loungewear using fabrics that meet such demands. Hence we suggest the necessity and effectiveness of loungewear made with environment-friendly hanji-fabric. 
Table 6. Textile surface designs in color groups

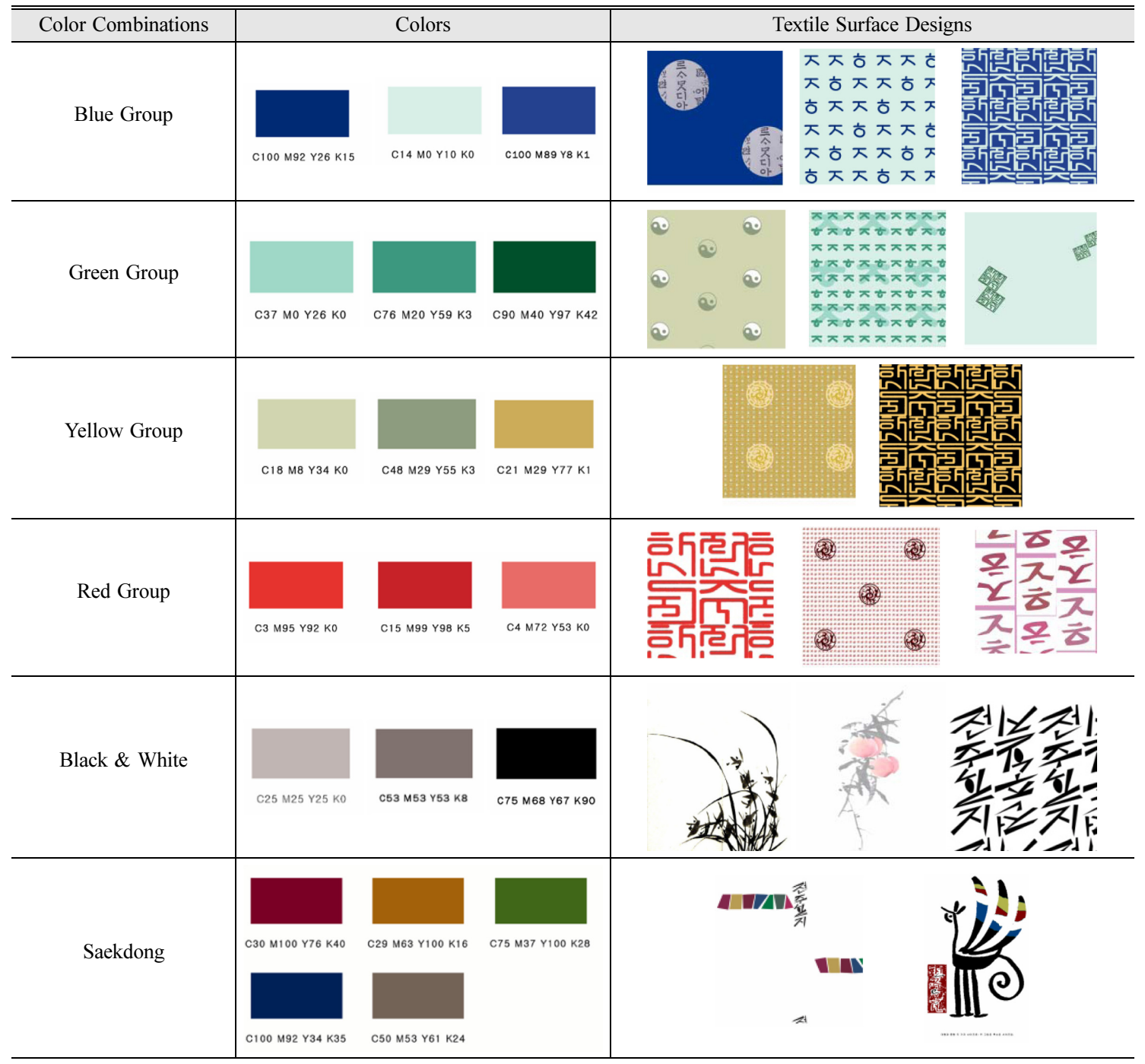

The main colors for loungewear were selected based on survey: blue, red, white, and saekdong (color stripes). We made color combinations with the main colors then applied the textile surface designs. The traditional Korean patterns of Hangeul (Korean letters), taegeuk (yin-yang), geometric figures, and samjogo (three-legged crow) were also chosen, and then loungewear using the textile designs was developed.

Suggestions of this research are as follows:

First, traditional Korean clothing has value as an important tool to spread Korean culture in this day and age where diverse cultures co-exist. Moderniza- tion of hanbok with an emphasis on practicality is needed to improve its acceptance to the public to make it a representative item that can promote traditional Korean culture to the world.

Second, the efforts of many fashion-related professionals are called on to promote Korean style loungewear not only in Korea but also in the world. To break the stereotypes of Korean fashion, the methods and practices of Korean fashion should be objectively assessed to improve the design proactively, which will help make Korean clothing suitable for those living in the $21^{\text {st }}$ century. 
Table 7. Design results of Korean style loungewear

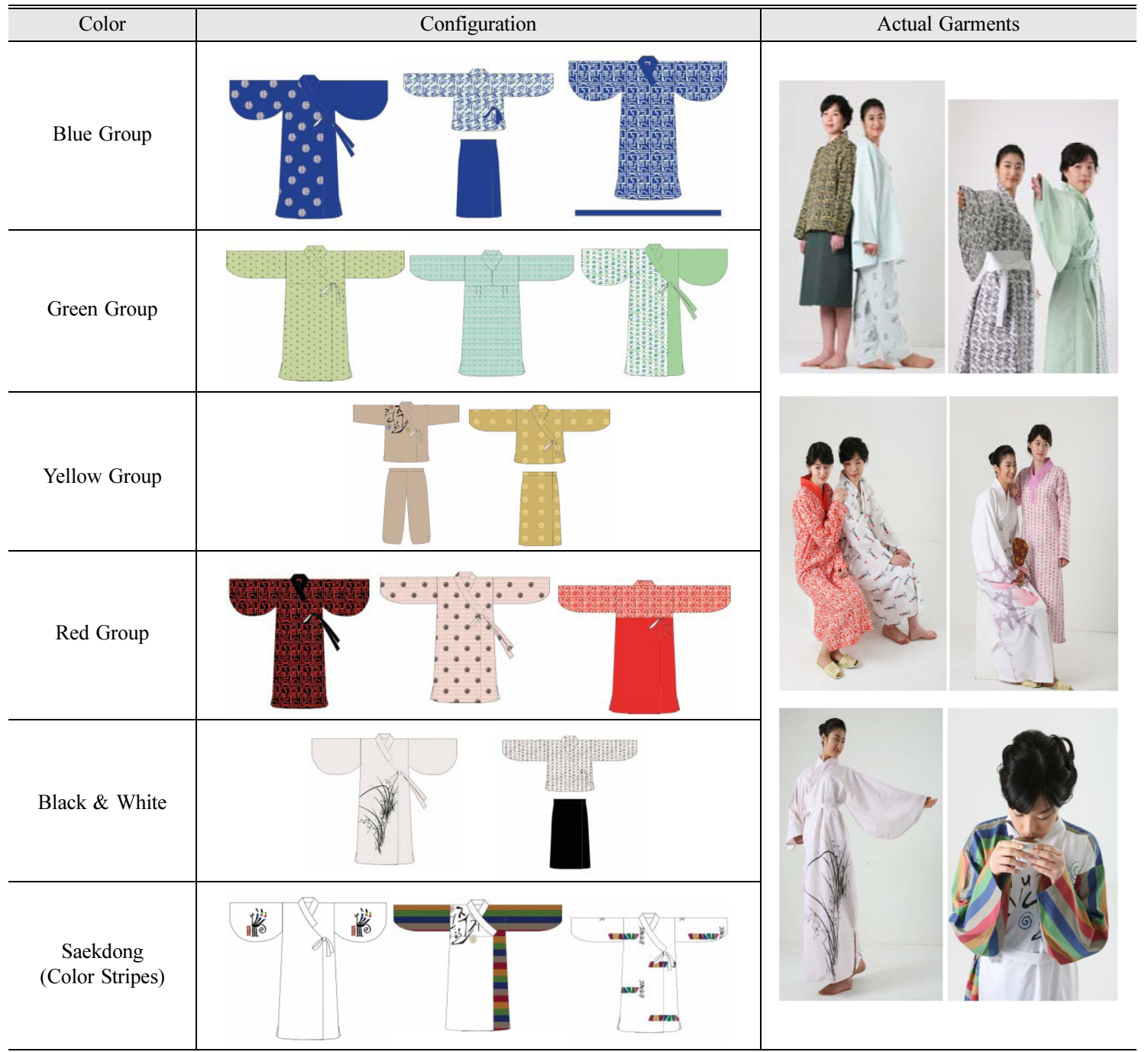

The results of the designs were exhibited in the Seoul Living Design Fair in March 2008 in COEX Hall in Seoul. The designs were also shown in "Hanstyle Fashion Show" at the Event Hall of Fashion Center in Seoul on April 7, 2008, conducted by the city of Jeonju, and subsequently exhibited in International Craft Trend Fair on December 3, 2008, in COEX Hall in Seoul.

\section{References}

Chae, K. S. (2001). Development of Korean style lounge- wear for tourist industry. Seoul: Jibmundang.

Chae, K. S. (2006). Korean jeogori 2000 years. Seoul: Sookmyung Women's University Press.

Chae, K. S. (2007). A study on improvement for globalization of hanbok-Focused on the successful cases in luxury fashion brands and Asian nations-. Journal of the Korean Society of Clothing and Textiles, 31(9/10), 1418-1430.

Chae, K. S., \& Koh, J. M. (2009). A study on the Baekje culture of costume (Part I). Journal of the Korean Society of Clothing and Textiles, 33(9), 1347-1360.

Fashion dictionary compilation committe. (1999). Fashion dictionary. Seoul: Kyomunsa. 